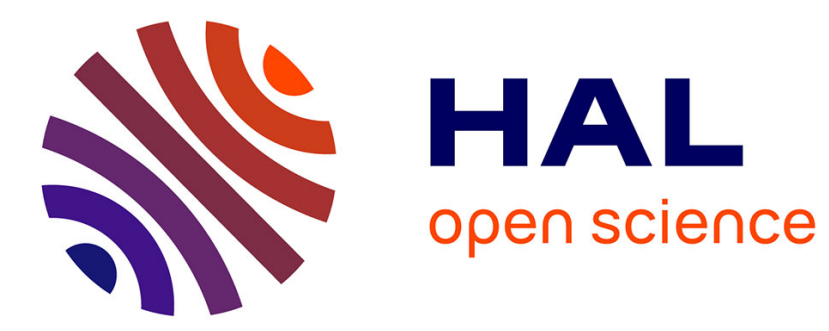

\title{
Le laboratoire international associé Bact-Inflam, l'aboutissement de 17 années de collaboration France-Brésil
}

Vasco Azevedo, Jean-Marc Chatel, Philippe Langella, Yves Le Loir

\section{- To cite this version:}

Vasco Azevedo, Jean-Marc Chatel, Philippe Langella, Yves Le Loir. Le laboratoire international associé Bact-Inflam, l'aboutissement de 17 années de collaboration France-Brésil. Histoire de la Recherche Contemporaine, 2018, https://journals.openedition.org/hrc/2490 (Tome VII N²), pp.197201. 10.4000/hrc.2490 . hal-02154535

\section{HAL Id: hal-02154535 \\ https://hal.science/hal-02154535}

Submitted on 12 Jun 2019

HAL is a multi-disciplinary open access archive for the deposit and dissemination of scientific research documents, whether they are published or not. The documents may come from teaching and research institutions in France or abroad, or from public or private research centers.
L'archive ouverte pluridisciplinaire HAL, est destinée au dépôt et à la diffusion de documents scientifiques de niveau recherche, publiés ou non, émanant des établissements d'enseignement et de recherche français ou étrangers, des laboratoires publics ou privés.

\section{(ㄷ)(1) $\$$}

Distributed under a Creative Commons Attribution - NonCommerciall 4.0 International 


\section{Le laboratoire international associé Bact-Inflam, l'aboutissement de 17 années de collaboration France-Brésil}

Vasco Azevedo, Jean-Marc Chatel, Philippe Langella et Yves Le Loir

\section{OpenEdition}

\section{Journals}

Édition électronique

URL : http://journals.openedition.org/hrc/2490

DOI : $10.4000 /$ hrc. 2490

ISSN : 2265-786X

\section{Éditeur}

CNRS Éditions

\section{Édition imprimée}

Date de publication : 1 novembre 2018

Pagination : 197-201

ISBN : 978-2-271-12426-5

ISSN : 2260-3875

Ce document vous est offert par INRA Institut National de la Recherche Agronomique

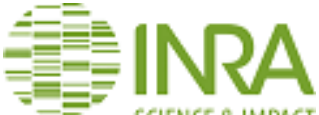 \\ SCIENCE \& IMPACT}

\section{Référence électronique}

Vasco Azevedo, Jean-Marc Chatel, Philippe Langella et Yves Le Loir, « Le laboratoire international associé Bact-Inflam, l'aboutissement de 17 années de collaboration France-Brésil », Histoire de la recherche contemporaine [En ligne], Tome VII N² | 2018, mis en ligne le 15 avril 2019, consulté le 14 mai 2019. URL : http://journals.openedition.org/hrc/2490 ; DOI : 10.4000/hrc.2490

Ce document a été généré automatiquement le 14 mai 2019.

(C) Comité pour l'histoire du CNRS 


\section{Le laboratoire international associé Bact-Inflam, l'aboutissement de 17 années de collaboration France- Brésil}

Vasco Azevedo

\section{Que sont les maladies inflammatoires et pourquoi constituent-elles un problème contemporain au Brésil et en France ?}

1 Les maladies inflammatoires regroupent diverses affections se caractérisant par l'inflammation d'un ou plusieurs organes. L'inflammation est un ensemble de réactions de défense générées par l'organisme en réponse à une agression. Deux types d'agression peuvent induire une inflammation: des agressions externes, telles que blessure, infection, traumatisme, ou internes à l'organisme lui-même comme dans certaines pathologies auto-immunes.

2 Le Brésil, comme la France, traverse une période de transition alimentaire profonde dont les répercussions commencent à se faire sentir en termes de santé publique. Ainsi, les dysfonctionnements digestifs d'origine inflammatoire prennent une ampleur croissante. De nombreuses pathologies humaines liées au mode de vie «à l'occidental ", obésité, diabète, allergie, maladies chroniques inflammatoires de l'intestin (MICI), sont caractérisées par une inflammation. Ce LIA offre donc un terrain de jeu intéressant (un pays occidental développé et un pays latino-américain en émergence) pour développer des projets de recherche et des alternatives thérapeutiques en santé humaine comme en santé animale répondant concrètement à une problématique internationale.

3 Concernant les aspects de santé animale (mammite) développés au sein du LIA, ils répondent eux aussi directement à des problématiques nationales fortes et s'inscrivent parfaitement dans une logique S3 (Smart Specialization Strategy) si l'on considère les implantations des laboratoires impliqués. L'État du Minas Gerais est en effet le plus gros 
producteur laitier du pays et est le seul état brésilien qui possède une réelle culture fromagère avec quelques $\mathrm{AOC}$ répertoriées. L'ouest de la France est aussi un bassin de production laitière important (le premier en France et deuxième en Europe). Rennes s'est imposé comme pôle d'excellence en recherche laitière et bénéficie d'un fort soutien régional. Or malgré d'indéniables efforts en conduite et suivi sanitaires des cheptels laitiers, les mammites (inflammation de la mamelle, le plus souvent d'origine infectieuse) demeurent un problème majeur pour la production dans ces deux régions. Elles induisent un coût et un surcroît de travail très important pour la France. Au Brésil, la piètre qualité des laits collectés constitue un frein au développement d'une industrie laitière performante dans le pays. Le LIA viendra donc s'inscrire dans un dispositif alliant, sur un même territoire, une industrie laitière, des bassins de productions laitières pourvoyeurs d'emplois et des programmes de recherche, d'enseignement et de formation professionnelle stimulant l'innovation et l'entreprenariat.

\section{Pourquoi s'intéresser à l'action des bactéries dans ce contexte?}

4 Dans chacun de ces contextes, intéressant la santé humaine ou animale, les bactéries liées à l'organe (intestin ou mamelle) jouent un rôle, soit en tant qu'éléments déclencheurs de l'inflammation, soit en tant qu'agents capables de moduler cette inflammation. Ainsi les bactéries pathogènes telles que Staphylococcus aureus sont-elles fréquemment impliquées dans les mammites chez les ruminants laitiers. S. aureus induit des infections souvent chroniques et, de ce fait, des inflammations récidiventes. Les mécanismes impliqués dans la pathogenèse de $S$. aureus et surtout dans la persistance des infections sont encore mal connus.

5 A l'opposé, certaines bactéries dites probiotiques sont capables de moduler l'inflammation au niveau du système digestif et de soulager certains des symptomes associés aux maladies inflammatoires de l'intestin. Plusieurs espèces de bactéries lactiques, dont Lactobacillus casei, constituent de bonnes candidates pour ces applications.

6 L'avènement des techniques de séquençage à haut débit a permis d'étudier en profondeur la composition en bactéries des écosystèmes intestinaux ou mammaires. Les premiers résultats montrent qu'à un statut sanitaire donné peut être associé une composition microbienne particulière. Ces études ont permis d'établir des profils de microbiotes en équilibre (eubiose) associés à un organe sain. Ceux-ci se caractérisent généralement par une forte biodiversité et sont dominés par des groupes bactériens particuliers. Ces études ont aussi permis de définir des microbiotes en déséquilibre (dysbiose) qui se caractérisent par une perte de diversité et l'absence (ou la très faible abondance) de certains groupes bactériens.

7 Mieux connaitre la composante bactérienne de ces maladies inflammatoires permettra de mieux comprendre les mécanismes inflammatoires et de proposer des méthodes basées sur l'utilisation de bactéries pour prévenir ou traiter ces maladies. Ceci devrait aider à réduire le recours aux traitements allopathiques (antibiotiques/antiinflammatoires). La réduction de l'usage d'antibiotiques à usage vétérinaire, notamment, est aujourd'hui une priorité mondiale afin d'éviter l'émergence de souches bactériennes résistantes aux antibiotiques et transmissibles de l'animal à l'homme. 


\section{Quels sont les parcours des acteurs du projet Bact-Inflam ?}

8 Philippe Langella, Yves Le Loir et Vasco Azevedo se connaissent depuis le début des années 1990, époque à laquelle Vasco Azevedo préparait une thèse de doctorat à l'Unité de Génétique Microbienne (UGM) à l'INRA de Jouy-en-Josas. Au cours de sa thèse encadrée par Pascale Serror (INRA), il avait pour mission de décrypter une partie du génome de Bacillus subtilis, bactérie à Gram positif modèle. C'est au cours de ces années en France, au contact de Dusko S. Ehrlich, directeur de l'UGM et un des pionniers de la génomique puis de la métagénomique bactérienne, qu'il a acquis la conviction que les génomes bactériens constituaient une source inépuisable de connaissances scientifiques. Il a pris aussi rapidement conscience de l'importance d'avoir les clés de décodage de ces génomes. En effet, au-delà des aspects techniques (on est, dans les années 1990, à une époque où la méthode de séquençage selon Sanger est fastidieuse et très coûteuse), Vasco Azevedo réalise rapidement tout l'intérêt de la science computationnelle, pas encore qualifiée de bio-informatique, pour l'analyse des données de séquençage. Après un séjour postdoctoral au département de microbiologie de la faculté de médecine de l'université de Pennsylvanie (Etats-Unis, 1994), il rentre au Brésil où il occupe d'abord un poste à l'université fédérale d'Ouro Preto (UFOP, à Ouro Preto, l'ancienne capitale de l'État du Minas Gerais) avant de partir à l'université fédérale du Minas Gerais (UFMG, à Belo Horizonte, la capitale actuelle de l'État du Minas Gerais) où il occupe désormais un poste de professeur titulaire. En écho à ses années de thèse en France, il a développé le premier cours de pos-graduação (master et doctorat) dédié à la bioinformatique au Brésil. Le programme de post-graduation de bioinformatique qu'il a créé est aujourd'hui classé 7 par la CAPES (ce qui est la note la plus haute et rarement attribuée, un synonyme d'excellence).

9 Sur cette même période, Yves Le Loir rejoint l'UGM pour préparer un DEA en parallèle de son diplôme d'ingénieur agronome (ENSA, Rennes). Il y prépare ensuite une thèse de doctorat en microbiologie (université Paris XI) à l'UGM, au sein de l'équipe d'Alexandra Gruss (INRA). Il était alors encadré par Philippe Langella (chargé de recherche INRA) dont il était le premier étudiant en thèse. De 1992 à 1996, ils développent conjointement des outils moléculaires pour l'étude de la sécrétion des protéines chez Lactococcus lactis, la bactérie lactique modèle. Ces études, pionnières dans l'utilisation biotechnologique des bactéries lactiques $(\mathrm{BL})$, perdurent dans l'équipe actuelle de $\mathrm{P}$. Langella. Après une période de recherche sur la respiration chez L. lactis (1999-2001), Yves Le Loir s'est installé en 2001 à Rennes, dans l'actuelle UMR STLO (Science \& Technologie du Lait \& de l'Oeuf) sur l'Agrocampus Ouest, pour initier une nouvelle thématique de recherche portant sur Staphylococcus aureus dans la filière laitière. Ses travaux ont porté sur l'adaptation à l'hôte ruminant et sur les interactions entre $S$. aureus et les BL qui l'ont amené à s'intéresser au contexte de la mammite, une inflammation de la mamelle souvent due à une infection bactérienne. Après avoir dirigé une équipe de recherche au sein du STLO (2008-2016) où était également étudié le potentiel probiotique de Propionibacterium freudenreichii (travaux de G. Jan), il est depuis 2017 directeur de l'UMR STLO .

Pendant cette même période, Philippe Langella a développé des travaux pionniers sur l'exploration du microbiote intestinal et de ses liens avec la santé de l'hôte. Il dirige une équipe, Interactions Commensales et Probiotiques avec l'Hôte (ProbiHôte), au sein de l'Institut MICALIS (Microbiologie de l'Alimentation au service de la Santé). En 2008, son 
équipe a identifié, sur la base de données cliniques humaines, la bactérie commensale anti-inflammatoire Faecalibacterium prausnitzii et a démontré son potentiel en utilisation thérapeutique pour prévenir et traiter des symptômes liés aux MICI. De nombreux travaux sur les capacités probiotiques de bactéries lactiques ou commensales de l'intestin ont hissé ProbiHôte parmi les meilleures équipes mondiales dans un domaine pourtant hautement compétitif. En attestent les nombreux brevets, publications et contrats de recherche en partenariat académique ou industriel. L'équipe de Philippe Langella s'est considérablement étoffée par les recrutements de L. Bermudez en 2006 (DR INRA) et de J.M. Chatel en 2008 (DR INRA). Elle comporte aujourd'hui une quarantaine de personnes réparties en 4 groupes dont ceux dirigés par L. Bermudez, "capacités probiotiques de bactéries intestinales et de nouveaux outils thérapeutiques et préventifs sous la forme de probiotiques recombinants ", et de J.-M. Chatel, "transfert d'ADN bactéries-hôte et immuno-modulation en contexte intestinal». Sous l'impulsion de P. Langella, L. Bermudez et J.-M. Chatel se sont particulièrement investis dans la coordination de projets franco-brésiliens.

11 Tout au long de ces années, Vasco Azevedo est resté attaché à la France et dès 1999, il obtient un financement de post-doctorat pour Luciana Ribeiro qui a été accueillie au sein de l'équipe de $P$. Langella pour une période d'un an. Les travaux ainsi initiés ont servi de base à l'élaboration d'un premier projet CAPES-COFECUB (Projet $N^{\circ} 319 / 00$ sur la période 2000-2004), porté par V. Azevedo et P. Langella. En 2001, malgré son départ pour Rennes, Y. Le Loir a continué à collaborer avec P. Langella et V. Azevedo. A l'issue du projet CAPES-COFECUB 319/00, les échanges ont continué pendant deux années (hors financement CAPES-COFECUB), notamment par la valorisation des résultats des étudiants brésiliens accueillis et la participation des collaborateurs français à des congrès brésiliens (Sociéte brésilienne de microbiologie et Société brésilienne de génétique). Ont suivi trois autres projets CAPES-COFECUB : le projet 359/06 (2006-2010) coordonné par Y. Le Loir et Sergio Costa ; le projet 720/11 (2011-2015), coordonné par Anderson Miyoshi (le premier doctorant brésilien accueilli en France et actuellement professeur assistant à l'UFMG) et par J.-M. Chatel (MICALIS) puis le projet 849/15 (période 2015-2019) actuellement coordonné par Ana Maria Caetano Faria (UFMG) et Luis Bermudez (MICALIS).

12 La synergie établie au sein de ce réseau de collaborations a permis aux équipes INRA de participer pleinement au programme Science sans Frontières (Ciência sem Fronteiras) lancé par le gouvernement brésilien en 2011. Les laboratoires INRA reçoivent désormais des étudiants financés par l'un ou l'autre de ces cadres. Par ailleurs, P. Langella et Y. Le Loir ont tous deux bénéficié du statut de Pesquisador Visitante Especial (PVE) à l'UFMG et ont développé, respectivement dans les laboratoires d'A. Miyoshi et V. Azevedo, des projets de recherche financés par le Brésil. Y. Le Loir a ainsi développé un projet de recherche «Estudo de quatro genes envolvidos na virulência de Staphylococcus aureus usando instrumentos de biologia molecular e abordagens ômicas » au sein du laboratoire LGCM de V. Azevedo. P. Langella a développé, quant à lui, un projet « Avaliação do efeito anti-inflamatório de duas linhagens de Lactococcus lactis contendo dois vetores de expressão eucariótica codificando a IL-4 ou a IL-10 murina para a prevenção de doenças inflamatórias intestinais » au sein du laboratoire d'A. Miyoshi.

13 Au cours de ces 18 années de collaboration, les chercheurs français ont effectué de nombreuses missions au Brésil, de deux à trois semaines chacune, auxquelles s'ajoutent deux missions de longue durée, effectuées par Y. Le Loir en 2012 et 2015. À chaque fois, ils ont eu l'occasion de participer à des séminaires dans plusieurs universités (UFMG-Belo 
Horizonte, USP-São Paulo et Piracicaba, UFP-Belém, UCB-Brasilia, UFF-Rio de Janeiro, UFV-Viçosa) et à plusieurs congrès (Société brésilienne de microbiologie, Société brésilienne de génétique, Société brésilienne d'immunologie, Journées de génétique des microorganismes, Congrès brésilien sur les pré-, pro-, symbiotiques) et divers workshops. Ils ont aussi pris part à la formation de base des étudiants de l'UFMG en donnant des cours de microbiologie moléculaire centrés sur la génétique des BL, sur les probiotiques et sur certains pathogènes de la filière laitière (notamment Staphylococcus aureus). En parallèle, pour améliorer ses interventions lors de ses séjours brésiliens, Y. Le Loir a suivi des cours de portugais à l'université de Rennes, de 2005 à 2010, et intervient désormais en portugais lors de ses séminaires au Brésil.

14 Ainsi, au cours de ces 18 années de collaborations, plus de 80 publications ont été cosignées dans des revues internationales à comité de lecture par des membres des équipes françaises et brésiliennes. A celles-ci s'ajoutent plusieurs articles de vulgarisation et chapitres de livre et de nombreuses communications à des conférences. Le STLO (Rennes) a accueilli onze doctorants brésiliens (dont dix en cotutelle entre Agrocampus Ouest et l'UFMG) et a été le premier laboratoire à établir une convention de cotutelle entre l'UFMG/ICB et l'INRA (thèse de P. Alves, soutenue en 2009). Par ailleurs, sept postdoctorants ont aussi séjourné au STLO. Depuis 2008, MICALIS a accueilli huit postdoctorants et deux thèses en cotutelle.

\section{Pourquoi un LIA et en quoi illustre-t-il la collaboration entre la France et le Brésil ?}

Le projet de recherche du LIA Bact-Inflam, porté par V. Azevedo, Y. Le Loir et J.-M. Chatel, vise à renforcer les liens entre l'INRA (les UMR STLO Rennes et MICALIS Jouy-en-Josas) et l'UFMG (ICB départements de biologie générale et de biochimie, Belo Horizonte) en renforçant et en formalisant les liens étroits unissant ces laboratoires. Deux équipes d'immunologie (du Pr M. Teixeira et du Pr A-C Caetano) ont rejoint ce consortium. Leurs expertises complètent opportunément celles développées au sein des trois équipes fondatrices et permettent d'étendre le champ des recherches aux mécanismes immunologiques sous-tendant les interactions bactéries-hôte.

Figure 1 mécanismes immunologiques sous-tendant les interactions bactéries-hôte

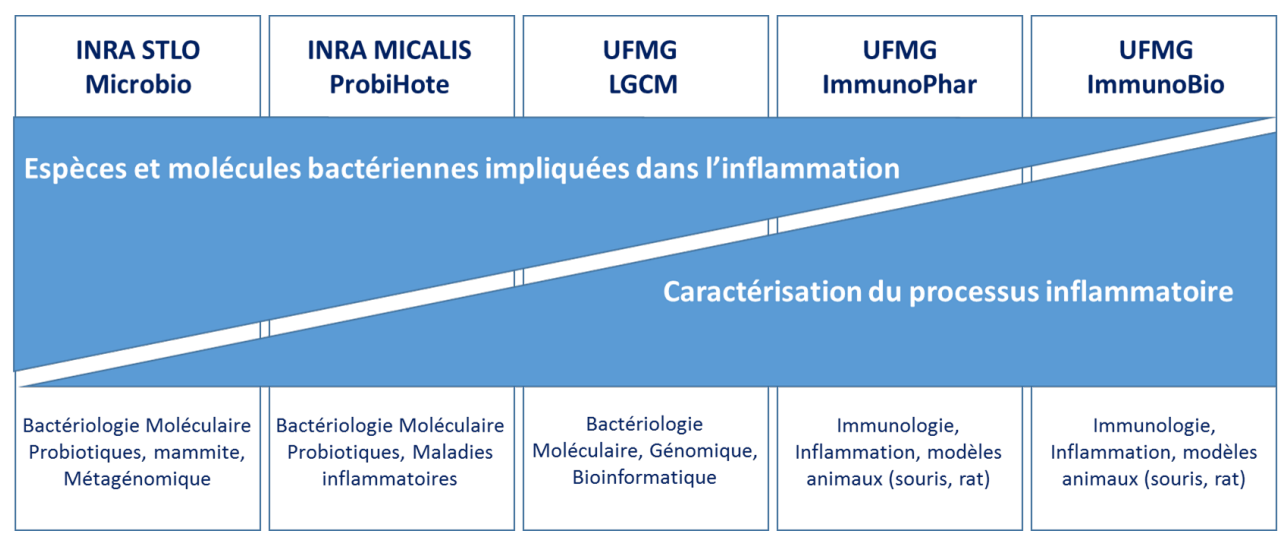

Du point de vue de l'INRA, la formalisation d'une collaboration via le label LIA prend en compte l'historique de la collaboration mais reconnait surtout l'excellence de la recherche scientifique menée en partenariat. Ce critère est le garant d'une collaboration 
symétrique et équilibrée. C'est aussi un gage de pérennité. Ce dernier point est un élément important de la démarche engagée par le consortium. Il s'agit, pour les coordinateurs $\mathrm{du}$ projet, de comuer une collaboration interpersonnelle en une collaboration institutionnelle engageant non plus quelques chercheurs mais l'ensemble de leurs équipes de recherche.

\section{Quel avenir pour le LIA?}

17 Au niveau scientifique, le projet vise à acquérir une meilleure compréhension du dialogue bactéries-bactéries au sein du microbiote et de l'impact du microbiote sur la biologie de l'hôte. Les échanges entre les laboratoires des deux institutions bonifieront la formation du personnel en leur donnant une perspective multidisciplinaire et internationale tout en perfectionnant leurs connaissances. En termes de santé publique et santé animale, il cherche à explorer de nouvelles approches thérapeutiques basées sur des stratégies de modulation du microbiote humain ou animal (pro- pré- et symbiotiques) contribuant in fine à réduire l'usage d'antibiotiques ou de traitement médicamenteux induits par les MICI ou les mammites. Les efforts menés dans ce sens sont encore très inégaux entre les deux pays. Le Brésil, comme la France, bénéficiera de cet effort qui produira une nouvelle génération de scientifiques, sensibilisés aux risques induits par ces maladies en matière de santé publique et formés à la recherche de solutions non allopathiques.

Enfin, au niveau institutionnel, le LIA permettra d'intensifier les échanges entre les deux institutions, au bénéfice des chercheurs et enseignents-chercheurs engagés mais aussi et surtout au bénéfice des jeunes scientifiques (doctorants et post-doctorants) formés par les laboratoires français et brésiliens. L'expérience à l'étranger offerte à de jeunes scientifiques à travers les séjours Brésil-France et France-Brésil leur ouvre de nouveaux horizons culturels et augmente leur employabilité. Le suivi professionnel des étudiants précédemment formés par nos laboraoires, hors LIA, nous montre à quel point cette expérience internationale leur a été profitable. Ces jeunes chercheurs sont autant de futurs cadres de la recherche académique ou privée. Gageons qu'au cours de leur carrière, quelle qu'elle soit, ils seront facilitateurs ou porteurs de nouveaux échanges scientifiques et ou commerciaux entre nos deux pays.

\section{RÉSUMÉS}

Le laboratoire international associé (LIA) Bact-Inflam, dédié à l'étude des bactéries et des maladies inflammatoires en santé humaine et animale, offre un exemple exceptionnel de coopération réussie entre le Brésil et la France depuis le début du XXI ${ }^{\mathrm{e}}$ siècle. 


\section{AUTEUR}

\section{VASCO AZEVEDO}

Professeur de génétique à l'université fédérale de Minas Gerais,

Jean-Marc Chatel, Philippe Langella et Yves Le Loir sont directeurs de recherche à l'Institut national de recherche agronomique (INRA)

Fr 